\title{
Robust Adaptive Beamforming Based on Worst-Case and Norm Constraint
}

\author{
Hongtao Li, ${ }^{1}$ Ke Wang, ${ }^{1}$ Chaoyu Wang, ${ }^{1,2}$ Yapeng He, ${ }^{3}$ and Xiaohua Zhu ${ }^{1}$ \\ ${ }^{1}$ Nanjing University of Science and Technology, Nanjing, Jiangsu 210094, China \\ ${ }^{2}$ China Shipbuilding Industry Corporation, Nanjing, Jiangsu 210003, China \\ ${ }^{3}$ China Academy of Space Technology, Xian, Shanxi 710000, China \\ Correspondence should be addressed to Ke Wang; xywangke@gmail.com
}

Received 24 October 2014; Accepted 9 December 2014

Academic Editor: Giampiero Lovat

Copyright (C) 2015 Hongtao Li et al. This is an open access article distributed under the Creative Commons Attribution License, which permits unrestricted use, distribution, and reproduction in any medium, provided the original work is properly cited.

A novel robust adaptive beamforming based on worst-case and norm constraint (RAB-WC-NC) is presented. The proposed beamforming possesses superior robustness against array steering vector (ASV) error with finite snapshots by using the norm constraint and worst-case performance optimization (WCPO) techniques. Simulation results demonstrate the validity and superiority of the proposed algorithm.

\section{Introduction}

As an important branch of array signal processing, adaptive beamforming technique has achieved a wide range of applications in the fields of radar, sonar, wireless communication, radio astronomy, and so forth [1]. However, adaptive beamforming confronts the problem of intense decrease in robustness in the cases that array steering vector (ASV) has errors, or receipt signal contains desired signal component, or the ideal covariance matrix is replaced by signal covariance matrix with finite snapshots [2]. It can be proven that the errors caused by using signal covariance matrix could be treated equally as the ASV errors at the circumstance of finite snapshots [3]. Therefore, the research of beamforming robustness primarily focuses on the ASV errors and the receipt signal containing desired signal.

In order to improve the adaptability of beamforming against those above situations, plenty of research on beamforming robustness has been carried out recently [4-16]. ESB algorithm possesses excellent robustness against ASV errors while its receipt signal must contain comparatively strong desired signal and prior information or estimation of the dimension of subspace is demanded [4]. Diagonal loading class-based adaptive beamforming algorithm possesses certain adaptability to the various situations while it is incapable of retaining the maximum gain to the actual desired signal and thus the signal to interference plus noise ratio (SINR) will, to some extent, suffer from loss with ASV errors [513]. Magnitude response constraints-based robust adaptive beamforming algorithm is blessed with favorable robustness against ASV errors by forming flat response in main beam while it demands prior information of main beam width and extra interference along with noise can be involved in the range of main beam [14-16].

To solve these problems, in this paper, a robust adaptive beamforming algorithm based on the worst-case and norm constraint (RAB-WC-NC) is proposed. RAB-WC-NC algorithm forms the flat response in the main beam width determined by the uncertainty set of ASV and improves the performance of beamforming by adopting norm constraint under the circumstance of finite snapshots. The proposed algorithm can improve the robustness of beamforming and suppress interference with finite snapshots.

\section{The Signal Model}

Consider a uniform linear array (ULA) with $N$ array elements separated from each other by a distance $d$. $M$ far-field narrowband incoherent signals are received from the orientation of $\left(\theta_{1}, \theta_{2}, \ldots, \theta_{M}\right)$, and then the receiving data of array $\mathbf{x}(t)$ can be expressed as

$$
\mathbf{x}(t)=\mathbf{A} \mathbf{s}(t)+\mathbf{n}(t),
$$


where $\mathbf{x}(t)=\left[x_{1}(t), x_{2}(t), \ldots, x_{N}(t)\right]^{T}, \mathbf{s}(t)=\left[s_{1}(t), s_{2}(t)\right.$, $\left.\ldots, s_{M}(t)\right]^{T}$ denote the observation vectors of array and signal at $t$ separately; $\mathbf{n}(t)=\left[n_{1}(t), n_{2}(t), \ldots, n_{N}(t)\right]^{T}$ is independent identically distributed gaussian white noise vector; $\mathbf{A}=$ $\left[\mathbf{a}\left(\theta_{1}\right), \mathbf{a}\left(\theta_{2}\right), \ldots, \mathbf{a}\left(\theta_{M}\right)\right]$ is the array manifold matrix;

$$
\begin{aligned}
\mathbf{a}\left(\theta_{m}\right)= & \frac{1}{\sqrt{N}} \\
& \cdot\left[1, e^{j 2 \pi d \sin \left(\theta_{m}\right) / \lambda}, \ldots, e^{j 2(N-1) \pi d \sin \left(\theta_{m}\right) / \lambda}\right]^{T}
\end{aligned}
$$

is the steering vector of signal at orientation $\theta_{m}$. Assume that the signal and noise are unrelated.

The output of array is the weight sum of the observation signals from each array element. The weight vector $\boldsymbol{\omega}=\left[\omega_{1}\right.$, $\left.\omega_{2}, \ldots, \omega_{N}\right]^{T}$, where $\omega_{k}$ denotes the $k$ th weight coefficient and the output of array is expressed as

$$
y(t)=\omega^{H} \mathbf{x}(t)
$$

The output power of array is presented as

$$
E\left\{y(t) y^{H}(t)\right\}=\boldsymbol{\omega}^{H} \mathbf{R}_{x} \boldsymbol{\omega},
$$

where $E\{\cdot\}$ denotes the mathematical expectation, and $\mathbf{R}_{x}=$ $E\left\{\mathbf{x}(t) \mathbf{x}^{H}(t)\right\}$ is the covariance matrix of array snapshot.

It is well known that MVDR beamforming minimizes array output power while constraining the desired signal response to be unity. That is,

$$
\begin{gathered}
\min \boldsymbol{\omega}^{H} \mathbf{R}_{x} \boldsymbol{\omega} \\
\text { subject to } \boldsymbol{\omega}^{H} \mathbf{a}\left(\theta_{0}\right)=1,
\end{gathered}
$$

where $\mathbf{a}\left(\theta_{0}\right)$ denotes the presumed ASV of the desired signal.

The weight vector of MVDR beamforming algorithm can be derived from Lagrange multiplier method:

$$
\boldsymbol{\omega}_{\mathrm{MVDR}}=\frac{\mathbf{R}_{x}^{-1} \mathbf{a}\left(\theta_{0}\right)}{\mathbf{a}\left(\theta_{0}\right)^{H} \mathbf{R}_{x}^{-1} \mathbf{a}\left(\theta_{0}\right)} .
$$

However, the presumed steering vector always deviates from the actual one. In this case, the performance of the MVDR beamformer is severely limited by target signal cancellation. To maintain a fairly stable gain in the region of interest, the following inequality constraints on the steering vector are imposed [6]:

$$
\begin{aligned}
& \min \boldsymbol{\omega}^{H} \mathbf{R}_{x} \boldsymbol{\omega} \\
& \text { subject to }\left|\boldsymbol{\omega}^{H} \widetilde{\mathbf{a}}\left(\theta_{0}\right)\right| \geq 1, \quad \forall\left\|\widetilde{\mathbf{a}}\left(\theta_{0}\right)-\mathbf{a}\left(\theta_{0}\right)\right\|_{2} \leq \varepsilon_{0},
\end{aligned}
$$

where $\varepsilon_{0}$ denotes the uncertainty factors of ASV and $\widetilde{\mathbf{a}}\left(\theta_{0}\right)$ denotes the actual ASV.

WCPO algorithm can be achieved by analyzing constraint condition and making the optimal performance of beamforming on the worst case:

$$
\begin{aligned}
& \min \boldsymbol{\omega}^{H} \mathbf{R}_{x} \boldsymbol{\omega} \\
& \text { subject to } \boldsymbol{\omega}^{H} \mathbf{a}\left(\theta_{0}\right) \geq 1+\varepsilon_{0}\|\boldsymbol{\omega}\|_{2} .
\end{aligned}
$$

Equation (8) can be transformed to SOCP problem, to be solved by internal point method (IPM) algorithm. And then this algorithm is deduced further to reduce the computational complexity by general iterative method [6].

\section{The Proposed Algorithm}

In this section, we propose a robust beamformer with the worst case performance optimization and the norm constraint. We can formulate the constrained robust problem as

$$
\begin{array}{ll}
\min & \boldsymbol{\omega}^{H} \mathbf{R}_{x} \boldsymbol{\omega} \\
\text { subject to } & \left|\boldsymbol{\omega}^{H} \widetilde{\mathbf{a}}\left(\theta_{0}\right)\right| \geq 1, \quad \forall\left\|\widetilde{\mathbf{a}}\left(\theta_{0}\right)-\mathbf{a}\left(\theta_{0}\right)\right\|_{2} \leq \varepsilon_{0} \\
& \|\boldsymbol{\omega}\|_{2} \leq \zeta_{0} .
\end{array}
$$

Meanwhile, the physical meaning of the constraint in WCPO algorithm, demonstrated in (7), is to ensure the output gain of beamforming no less than unity within the error range of ASV, or in other words to form flat response within the main beam. Therefore, (9) can be transformed to

$$
\begin{array}{ll}
\min & \boldsymbol{\omega}^{H} \mathbf{R}_{x} \boldsymbol{\omega} \\
\text { subject to } & \left|\boldsymbol{\omega}^{H} \mathbf{a}(\theta)-\boldsymbol{\omega}_{M}^{H} \mathbf{a}(\theta)\right| \leq \delta, \quad \theta \in\left[\theta_{L}, \theta_{U}\right] \\
& \|\boldsymbol{\omega}\|_{2} \leq \zeta_{0} \\
& \left\|\widetilde{\mathbf{a}}\left(\theta_{0}\right)-\mathbf{a}\left(\theta_{0}\right)\right\|_{2} \leq \varepsilon_{0},
\end{array}
$$

where $\left[\theta_{L}, \theta_{U}\right]$ denotes the main beam width, $\delta$ denotes the ripple of main beam, and $\omega_{M}$ denotes the weight vector of expected flat response beamforming.

From the definition of $\mathbf{a}\left(\theta_{l}\right)$, we know that

$$
\left\|\mathbf{a}^{H}\left(\theta_{0}\right)\right\|_{2}=1 \text {. }
$$

Using the 3 rd constraint of (10), then

$$
\begin{aligned}
\left\|\widetilde{\mathbf{a}}\left(\theta_{0}\right)\right\|_{2} & =\left\|\left(\widetilde{\mathbf{a}}\left(\theta_{0}\right)-\mathbf{a}\left(\theta_{0}\right)\right)+\mathbf{a}\left(\theta_{0}\right)\right\|_{2} \\
& \leq\left\|\left(\widetilde{\mathbf{a}}\left(\theta_{0}\right)-\mathbf{a}\left(\theta_{0}\right)\right)\right\|_{2}+\left\|\mathbf{a}\left(\theta_{0}\right)\right\|_{2} \\
& \leq \varepsilon_{0}+1 .
\end{aligned}
$$

By using the constraint again, hence we have

$$
\begin{aligned}
1 & =\left\|\mathbf{a}\left(\theta_{0}\right)\right\|_{2} \\
& =\left\|\left(\mathbf{a}\left(\theta_{0}\right)-\widetilde{\mathbf{a}}\left(\theta_{0}\right)\right)+\widetilde{\mathbf{a}}\left(\theta_{0}\right)\right\|_{2} \\
& \leq\left\|\left(\mathbf{a}\left(\theta_{0}\right)-\widetilde{\mathbf{a}}\left(\theta_{0}\right)\right)\right\|_{2}+\left\|\widetilde{\mathbf{a}}\left(\theta_{0}\right)\right\|_{2} \\
& \leq \varepsilon_{0}+\left\|\widetilde{\mathbf{a}}\left(\theta_{0}\right)\right\|_{2} .
\end{aligned}
$$

Thus according to (12) and (13), we can get

$$
1-\varepsilon_{0} \leq\left\|\widetilde{\mathbf{a}}\left(\theta_{0}\right)\right\|_{2} \leq 1+\varepsilon_{0} .
$$


Multiplying the constant $\left\|\mathbf{a}^{H}\left(\theta_{0}\right)\right\|_{2}$ in both sides of the 3 rd constraint of (10),

$$
\begin{aligned}
& \left\|\mathbf{a}^{H}\left(\theta_{0}\right) \widetilde{\mathbf{a}}\left(\theta_{0}\right)-\mathbf{a}^{H}\left(\theta_{0}\right) \mathbf{a}\left(\theta_{0}\right)\right\|_{2} \\
& \quad \leq\left\|\mathbf{a}^{H}\left(\theta_{0}\right)\right\|_{2} \cdot\left\|\widetilde{\mathbf{a}}\left(\theta_{0}\right)-\mathbf{a}\left(\theta_{0}\right)\right\|_{2} \\
& \quad \leq\left\|\mathbf{a}^{H}\left(\theta_{0}\right)\right\|_{2} \cdot \varepsilon_{0} .
\end{aligned}
$$

Since $\left\|\mathbf{a}^{H}\left(\theta_{0}\right)\right\|_{2}=1$, then

$$
\left\|\mathbf{a}^{H}\left(\theta_{0}\right) \widetilde{\mathbf{a}}\left(\theta_{0}\right)-1\right\|_{2} \leq \varepsilon_{0} .
$$

According to the property of vector norm,

$$
\left|\left\|\mathbf{a}^{H}\left(\theta_{0}\right) \widetilde{\mathbf{a}}\left(\theta_{0}\right)\right\|_{2}-1\right| \leq\left\|\mathbf{a}^{H}\left(\theta_{0}\right) \widetilde{\mathbf{a}}\left(\theta_{0}\right)-1\right\|_{2} \leq \varepsilon_{0} .
$$

After simplification,

$$
1-\varepsilon_{0} \leq\left\|\mathbf{a}^{H}\left(\theta_{0}\right) \widetilde{\mathbf{a}}\left(\theta_{0}\right)\right\|_{2} \leq 1+\varepsilon_{0} .
$$

According to the symmetry of signal in both space and frequency domains, if $\widetilde{\mathbf{a}}\left(\theta_{0}\right)$ is treated as a "signal" with $N$ point time sequence, then the physical meaning of (14) is that the energy interval of signal $\widetilde{\mathbf{a}}\left(\theta_{0}\right)$ is $\left[1-\varepsilon_{0}, 1+\varepsilon_{0}\right]$. Taking $\mathbf{a}\left(\theta_{0}\right)$ as the coefficient of $N$-order filter, then the filter is a bandpass one with center frequency being $\theta_{0}$. Since $\left\|\mathbf{a}^{H}\left(\theta_{0}\right)\right\|_{2}=$ 1 , the maximum gain of the filter is unity. Combining the physical meaning of (14) and (18), the energy interval of output signal is $\left[1-\varepsilon_{0}, 1+\varepsilon_{0}\right]$ after the signal $\widetilde{\mathbf{a}}\left(\theta_{0}\right)$ goes through band-pass filter, of which maximum gain is unity.

The position of peak value of "signal" $\widetilde{\mathbf{a}}\left(\theta_{0}\right)$ in frequency domain corresponds to the position of main beam in space domain. Consider the worst case when the energy of input "signal" $\widetilde{\mathbf{a}}\left(\theta_{0}\right)$ is $1+\varepsilon_{0}$ while the output energy of signal through the filter is $1-\varepsilon_{0}$. So the maximum attenuation of signal through filter in frequency domain is $\left(1-\varepsilon_{0}\right) /\left(1+\varepsilon_{0}\right)$. Assume that the attenuation of filter is equal to or less than $\left(1-\varepsilon_{0}\right) /\left(1+\varepsilon_{0}\right)$, and the corresponding frequency range is $\left[f_{L}, f_{U}\right]$, so the main peak of "signal" $\widetilde{\mathbf{a}}\left(\theta_{0}\right)$ must drop in the range of $\left[f_{L}, f_{U}\right]$.

Assume that the corresponding angle of ASV of real main beam in space domain is $\widehat{\theta}_{0}$; then the value interval of $\sin \left(\widehat{\theta}_{0}\right)$ is $\left[f_{L}, f_{U}\right]$, according to the symmetry of space domain and frequency domain. Therefore, the real range of main beam $\left[\theta_{L}, \theta_{U}\right]$ can be easily obtained by solving the arc-sin function.

So, the parameters of main beam width, $\theta_{L}$ and $\theta_{U}$, can be achieved by solving

$$
\left\|\widetilde{\mathbf{a}}^{H}\left(\widehat{\theta}_{0}\right)\right\|_{2}=\frac{\left(1-\varepsilon_{0}\right)}{\left(1+\varepsilon_{0}\right)} .
$$

At last, amending (10) using the main beam width information obtained from (19), RAB-WC-NC algorithm can be easily drawn:

$$
\begin{array}{ll}
\min & \boldsymbol{\omega}^{H} \mathbf{R}_{x} \boldsymbol{\omega} \\
\text { subject to } & \left|\boldsymbol{\omega}^{H} \mathbf{a}(\theta)-\boldsymbol{\omega}_{M}^{H} \mathbf{a}(\theta)\right| \leq \delta, \quad \theta \in\left[\theta_{L}, \theta_{U}\right] \\
& \|\boldsymbol{\omega}\|_{2} \leq \zeta_{0} \\
& \left\|\mathbf{a}^{H}\left(\theta_{i}\right)\right\|_{2}=\frac{\left(1-\varepsilon_{0}\right)}{\left(1+\varepsilon_{0}\right)}, \quad \theta_{i}=\theta_{L}, \theta_{U} .
\end{array}
$$

Using the Cholesky decomposition, covariance matrix of array snapshot can be given as

$$
\mathbf{R}_{x}=\mathbf{V}^{H} \mathbf{V} .
$$

Hence, (4) can be transformed to

$$
\boldsymbol{\omega}^{H} \mathbf{R}_{x} \boldsymbol{\omega}=(\mathbf{V} \boldsymbol{\omega})^{H}(\mathbf{V} \boldsymbol{\omega})=\|\mathbf{V} \boldsymbol{\omega}\|_{2}^{2} .
$$

Thus, (20) can be rewritten as

$\min \quad \eta$

subject to $\left|\boldsymbol{\omega}^{H} \mathbf{a}(\theta)-\boldsymbol{\omega}_{M}^{H} \mathbf{a}(\theta)\right| \leq \delta, \quad \theta \in\left[\theta_{L}, \theta_{U}\right]$

$$
\begin{aligned}
& \|\boldsymbol{\omega}\|_{2} \leq \zeta_{0} \\
& \|\mathbf{V} \boldsymbol{\omega}\|_{2} \leq \eta \\
& \left\|\mathbf{a}^{H}\left(\theta_{i}\right)\right\|_{2}=\frac{\left(1-\varepsilon_{0}\right)}{\left(1+\varepsilon_{0}\right)}, \quad \theta_{i}=\theta_{L}, \theta_{U} .
\end{aligned}
$$

As mentioned before, (23) can be solved by IPM algorithm as well.

In conclusion, the step of RAB-WC-NC algorithm can be generalized as follows.

Worst-Case and Norm Constraint-Based Robust Adaptive Beamforming Algorithm

Step 1. Use (19) to calculate the main beam width $\left[\theta_{L}, \theta_{U}\right]$.

Step 2. Adopt IPM algorithm to solve (24) to obtain the RABWC-NV algorithm weight vector:

$$
\begin{array}{ll}
\min & \eta \\
\text { subject to } & \left|\boldsymbol{\omega}^{H} \mathbf{a}(\theta)-\boldsymbol{\omega}_{M}^{H} \mathbf{a}(\theta)\right| \leq \delta, \quad \theta \in\left[\theta_{L}, \theta_{U}\right] \\
& \|\boldsymbol{\omega}\|_{2} \leq \zeta_{0} \\
& \|\mathbf{V} \boldsymbol{\omega}\|_{2} \leq \eta .
\end{array}
$$

\section{Simulation}

In our simulations, a ULA of $N=32$ array elements spaced a half wavelength apart is used. Desired signal and interference are both far-field narrow-band signal; the additive noise is 


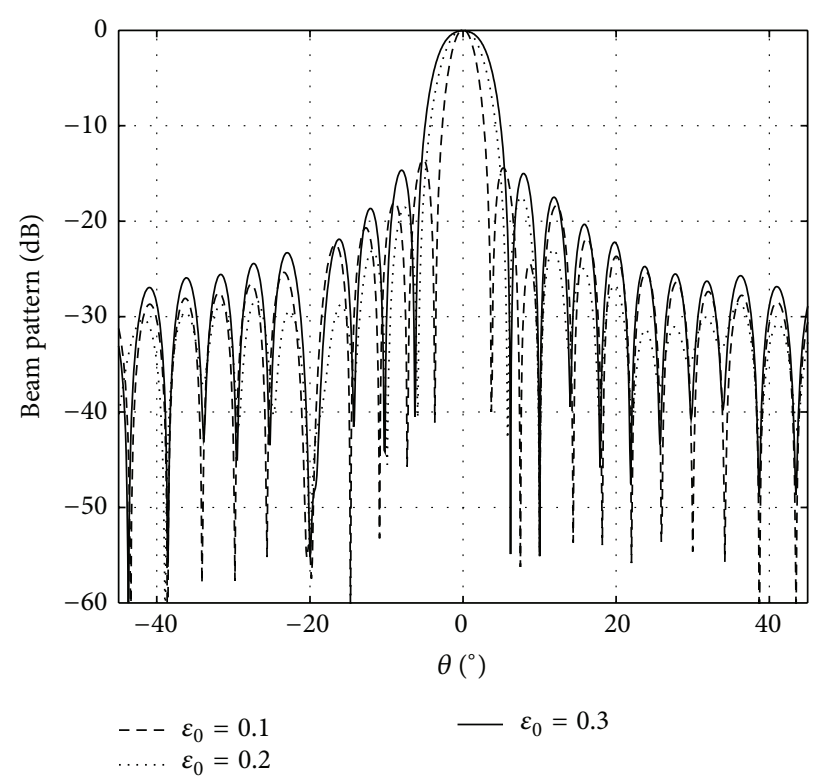

(a) Beam patterns

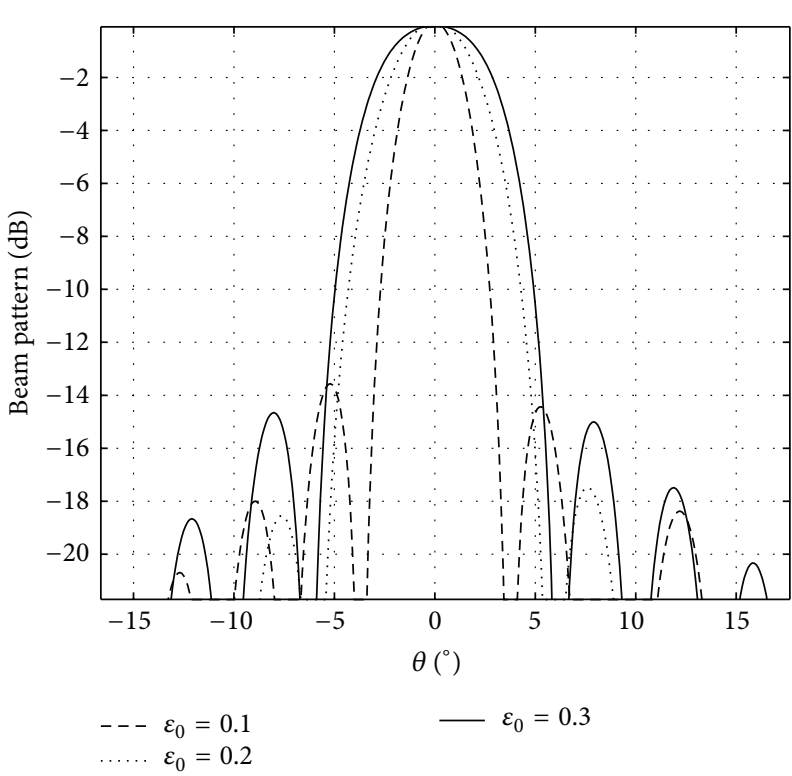

(b) Main beams

Figure 1: Various beamformers' beam patterns with $20 \times N$ snapshots.

modeled as a complex circularly symmetric Gaussian zeromean spatially and temporally white process. RAB-WC$\mathrm{NC}$ algorithm is compared with WCPO, norm constraint Capon beamforming (NCCB), SMI and LSMI algorithm with different snapshots, input SNR, and errors of ASV.

Example 1 (RAB-WC-NC algorithm with different error upper bonds and sufficient snapshots). We assume that the orientation of desired signal is $0^{\circ}$, SNR of signal is $10 \mathrm{~dB}$, and directions of arrival (DOA) of two interferences are $-20^{\circ}$, $10^{\circ}$ separately, the INR of which is $40 \mathrm{~dB}$, while snapshots are $20 \times N=640$. Figure 1(a) shows the beam patterns of RAB-WC-NC algorithm with different error upper bonds $\varepsilon_{0}$. As illustrated in Figure 1(a), RAB-WC-NC algorithm can still form effective beams when the receipt signal contains comparatively strong desired signal. With the increase of $\varepsilon_{0}$, the main beam width in the beam patterns of RAB-WC$\mathrm{NC}$ algorithm widens accordingly and forms nulls in several interference points, the depth of which can be $-55 \mathrm{~dB}$, satisfying the requirement of interference suppression. Figure $1(b)$ shows the main beam of RAB-WC-NC algorithm.

Example 2 (RAB-WC-NC algorithm with different error upper bonds and finite snapshots). As illustrated in Figure 2(a), with the decrease of snapshots, nulls produced by RAB-WC-NC algorithm with different main beam widths become slightly shallower while the depth of them still meets the requirement of interference suppression. Figure 2(b) shows the main beam of RAB-WC-NC algorithm.

Example 3 (beam patterns with high SNR). We assume that the orientation of desired signal is $0^{\circ}$, orientation of actual desired signal is $0.3^{\circ}$, SNR of signal is $30 \mathrm{~dB}$, DOAs of two interferences are $-20^{\circ}, 10^{\circ}$ separately, the INR of which is
$40 \mathrm{~dB}$, the error upper bond $\varepsilon_{0}=0.3$, and LNR of LSMI algorithm is $10 \mathrm{~dB}$. Figure $3(\mathrm{a})$ demonstrates the comparison among the beam patterns of RAB-WC-NC algorithm, NCCB algorithm, SMI algorithm, and LSMI algorithm at the snapshots of $2 \times N=64$. As shown in Figure 3(b), the array gain of RAB-WC-NC algorithm at $0.3^{\circ}$ loses $0.02 \mathrm{~dB}$ compared to it at $0^{\circ}$, but the gain of desired signal suffers almost no loss. In contrast, the array gain of NCCB algorithm in the direction of $0.3^{\circ}$ loses more than $1.5 \mathrm{~dB}$ compared to the maximum one and the SMI algorithm and the LMSI algorithm both produce nulls in the direction of $0.3^{\circ}$. Figure $3(\mathrm{~b})$ shows the comparison among the beam patterns of RAB-WC-NC algorithm, NCCB algorithm, SMI algorithm, and LSMI algorithm at the snapshots $2 \times N=64$. From Figure 3(b), it can be seen that the depth of nulls of RAB-WC-NC algorithm with finite snapshots tends to be slightly smaller than it with sufficient snapshots, which nevertheless can still meet the requirement of interference suppression; NCCB algorithm, SMI algorithm, and LSMI algorithm all share the phenomena that the depth of nulls becomes smaller and the sidelobe tends to be higher, leading to the degradation of performance.

Example 4 (output SINR with different error upper bonds). We assume that DOAs of two interferences are $-20^{\circ}, 10^{\circ}$ separately, the INR of which is $50 \mathrm{~dB}$, SNR of signal is $10 \mathrm{~dB}$, the snapshots are $20 \times N=640$, and 500 times of Monte Carlo experiments have been done. Figure 4 shows the output SINR versus different error upper bond $\varepsilon_{0}$, of RAB-WCNC algorithm, WCPO algorithm, NCCB algorithm, SMI algorithm, and LSMI algorithm. From Figure 4, it can be seen that the output SINR of RAB-WC-NC algorithm remains basically unchanged with the increase of error upper bond $\varepsilon_{0}$, which means that RAB-WC-NC algorithm possesses outstanding adaptability against the ASV errors. The output 


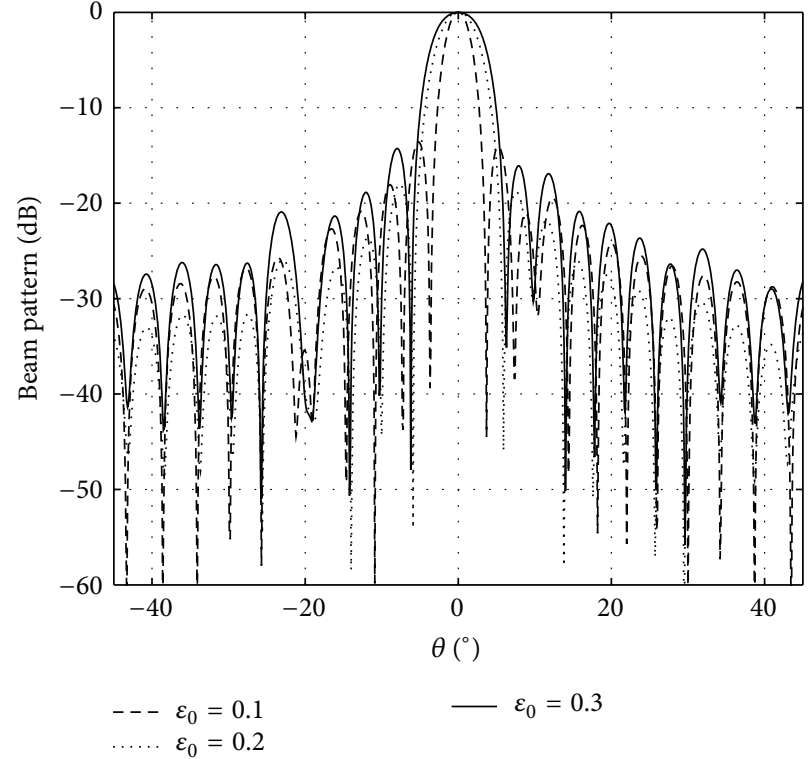

(a) Beam patterns

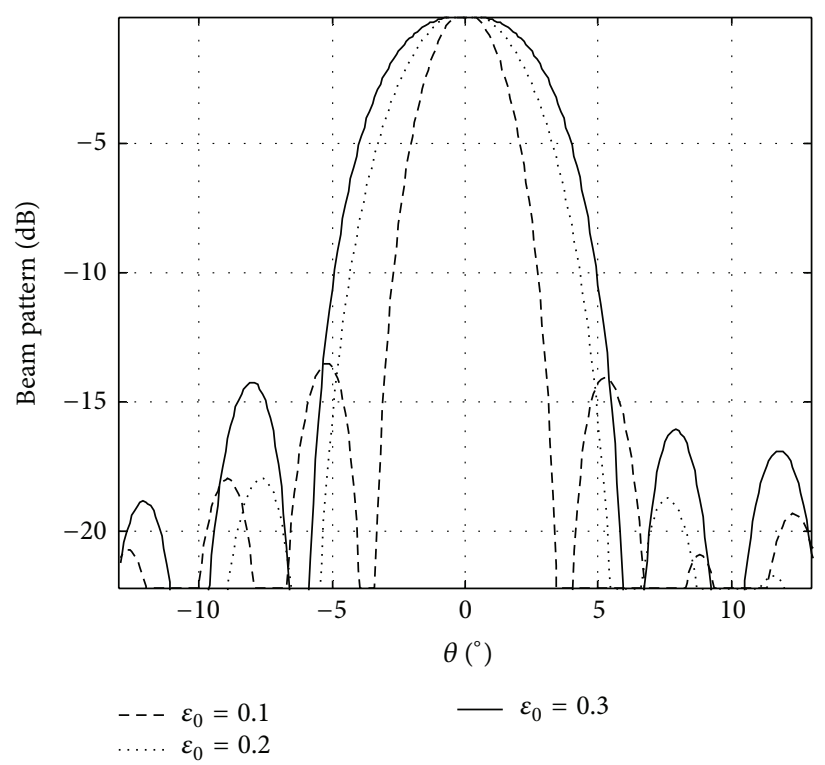

(b) Main beams

FIGURE 2: Various beamformers' beam patterns with $2 \times N$ snapshots.

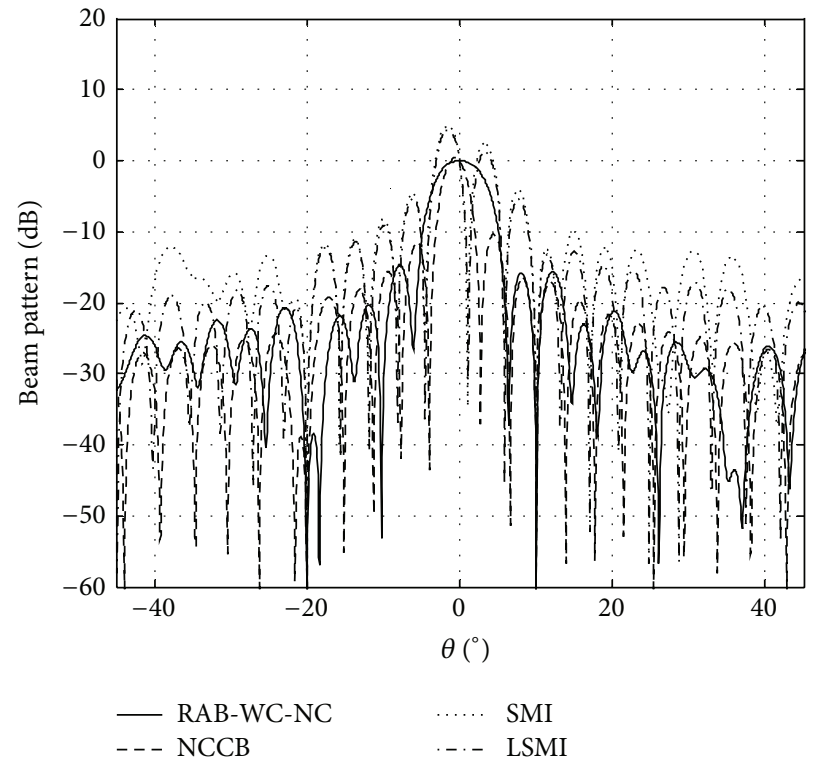

(a) $20 \times N$ snapshots

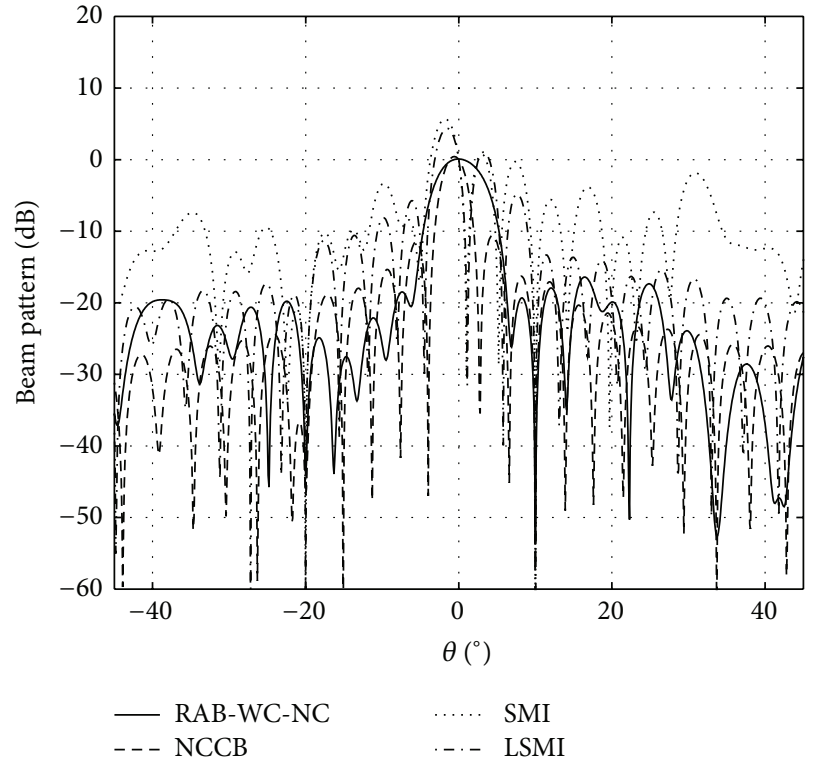

(b) $2 \times N$ snapshots

FIGURE 3: Various beamformers' beam patterns with high SNR.

SINR of NCCB algorithm and WCPO algorithm slightly decrease with the increase of error upper bond $\varepsilon_{0}$ because they cannot retain the maximum gain to desired signal. Due to the appearance of target signal cancellation, the output SINR of SMI algorithm and LSMI algorithm decrease drastically with the increase of error upper bond $\varepsilon_{0}$. Due to the diagonal loading, the performance of LSMI algorithm slightly exceeds that of SMI.
Example 5 (output SINR with different input SNR). We assume that DOAs of two interferences are $-10^{\circ}, 20^{\circ}$ separately, the INR of which is $50 \mathrm{~dB}$, the DOA of desired signal is $1^{\circ}$, the snapshots are $20 \times N=640$, and 500 times of Monte Carlo experiments have been done. Figure 5 shows the output SINR versus different input SNR, of RAB-WCNC algorithm, WCPO algorithm, NCCB algorithm, SMI algorithm, and LSMI algorithm. From Figure 5, it can be 


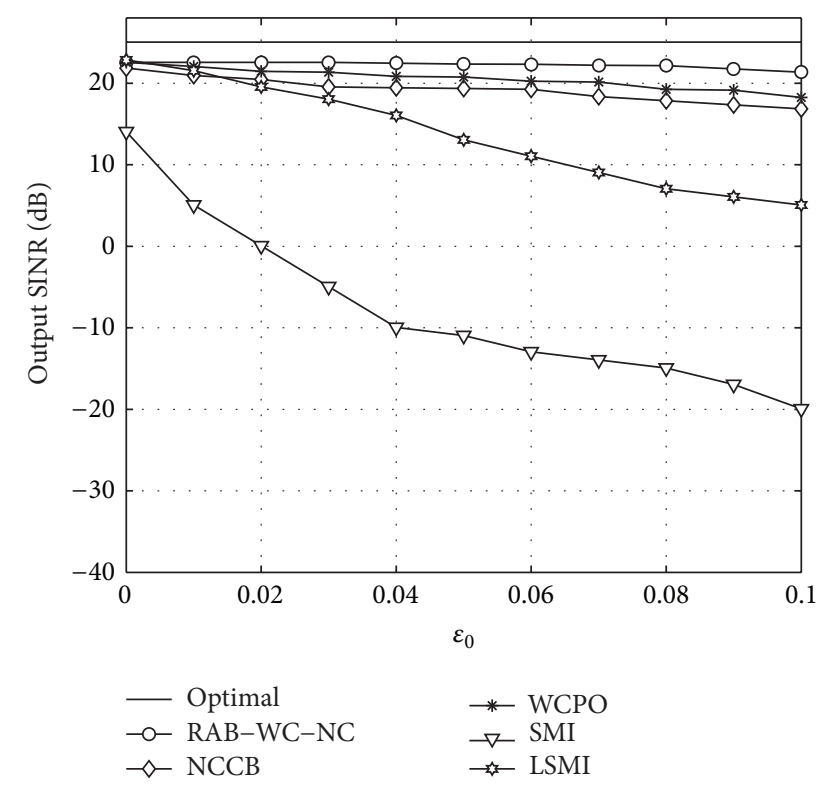

FIgURE 4: Output SINR versus $\varepsilon_{0}$.

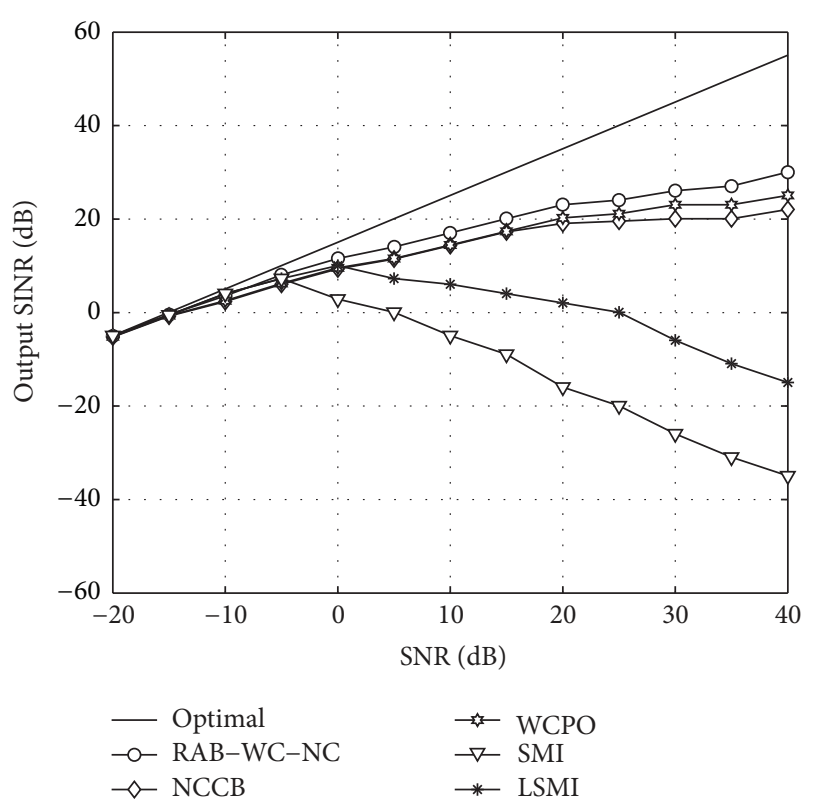

FIgURE 5: Output SINR versus input SNR.

seen that the performances of the proposed beamformers are always close to the optimal SINR in a large range from $-20 \mathrm{~dB}$ to $40 \mathrm{~dB}$. Furthermore, the proposed algorithms enjoy much faster convergence rates than others.

\section{Conclusion}

RAB-WC-NC algorithm is deduced specifically to handle the situations where ASV has errors, and the receipt data contains desired signal with finite snapshots. The uncertain set of ASV is adopted to determine the main beam width, within which the flat response is formed, increasing the robustness of beamforming against ASV error; and the performance of the proposed algorithm in the situations that receipt data contains desired signal with finite snapshots is improved by utilizing norm constraint. To sum up, RAB-WC-NC algorithm is blessed with certain adaptability to any kind of errors and effectively increases the output SINR under the circumstance of various errors, consequently proven to be a robust adaptive beamforming algorithm.

\section{Conflict of Interests}

The authors declare that there is no conflict of interests regarding the publication of this paper.

\section{Acknowledgment}

This work was supported by the National Natural Science Foundation of China under Grant 61401204.

\section{References}

[1] J. Li and P. Stoica, Robust Adaptive Beamforming, Wiley, New York, NY, USA, 2005.

[2] Y. Huang, Q. Li, W.-K. Ma, and S. Zhang, "Robust multicast beamforming for spectrum sharing-based cognitive radios," IEEE Transactions on Signal Processing, vol. 60, no. 1, pp. 527533, 2012.

[3] D. D. Feldman and L. J. Griffiths, "Projection approach for robust adaptive beamforming," IEEE Transactions on Signal Processing, vol. 42, no. 4, pp. 867-876, 1994.

[4] L. Chang and C.-C. Yeh, "Performance of DMI and eigenspacebased beamformers," IEEE Transactions on Antennas and Propagation, vol. 40, no. 11, pp. 1336-1347, 1992.

[5] H. Cox, R. Zeskind, and M. Owen, "Robust adaptive beamforming," IEEE Transactions on Acoustic, Speech and Signal Processing, vol. 35, no. 10, pp. 1365-1376, 1987.

[6] S. A. Vorobyov, A. B. Gershman, and Z.-Q. Luo, "Robust adaptive beamforming using worst-case performance optimization: a solution to the signal mismatch problem," IEEE Transactions on Signal Processing, vol. 51, no. 2, pp. 313-324, 2003.

[7] J. Li, P. Stoica, and Z. Wang, "On robust Capon beamforming and diagonal loading," IEEE Transactions on Signal Processing, vol. 51, no. 7, pp. 1702-1715, 2003.

[8] J. Li, P. Stoica, and Z. Wang, "Doubly constrained robust Capon beamformer," IEEE Transactions on Signal Processing, vol. 52, no. 9, pp. 2407-2423, 2004.

[9] S. Shahbazpanahi, A. B. Gershman, Z.-Q. Lou, and K. M. Wong, "Robust adaptive beamforming for general-rank signal models," IEEE Transactions on Signal Processing, vol. 51, no. 9, pp. 22572269, 2003.

[10] Y. Wang, H.-W. Liu, B. Jiu, and X.-C. Yang, "Robust transmitting beamforming for MIMO radar," Journal of Electronics \& Information Technology, vol. 34, no. 2, pp. 318-323, 2012.

[11] C.-Y. Chen and P. P. Vaidyanathan, "Quadratically constrained beamforming robust against direction-of-arrival mismatch," IEEE Transactions on Signal Processing, vol. 55, no. 8, pp. 41394150, 2007.

[12] M. Rübsamen and M. Pesavento, "Maximally robust capon beamformer," IEEE Transactions on Signal Processing, vol. 61, no. 8, pp. 2030-2041, 2013. 
[13] B.-B. Xie, L. Gan, and L.-P. Li, "Robust capon beamforming with orthomodular constraint," Journal of Electronics and Information Technology, vol. 33, no. 9, pp. 2045-2049, 2011.

[14] Z. L. Yu, W. Ser, M. H. Er, Z. Gu, and Y. Li, "Robust adaptive beamformers based on worst-case optimization and constraints on magnitude response," IEEE Transactions on Signal Processing, vol. 57, no. 7, pp. 2615-2628, 2009.

[15] D. Xu, R. He, and F. Shen, "Robust beamforming with magnitude response constraints and conjugate symmetric constraint," IEEE Communications Letters, vol. 17, no. 3, pp. 561-564, 2013.

[16] H.-T. Li, Y.-P. He, X.-H. Zhu, and W. Hu, "Spectral analysis based robust adaptive beamforming algorithm," Chinese Journal of Radio Science, vol. 27, no. 1, pp. 147-151, 2012. 

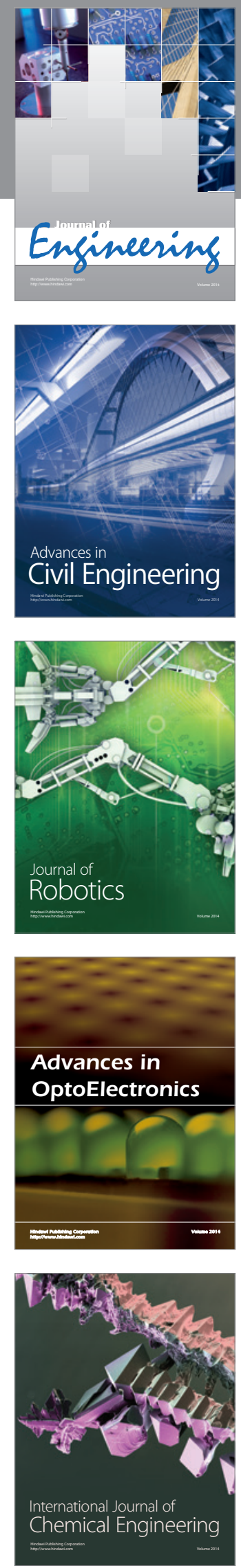

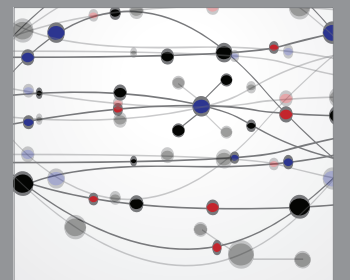

The Scientific World Journal
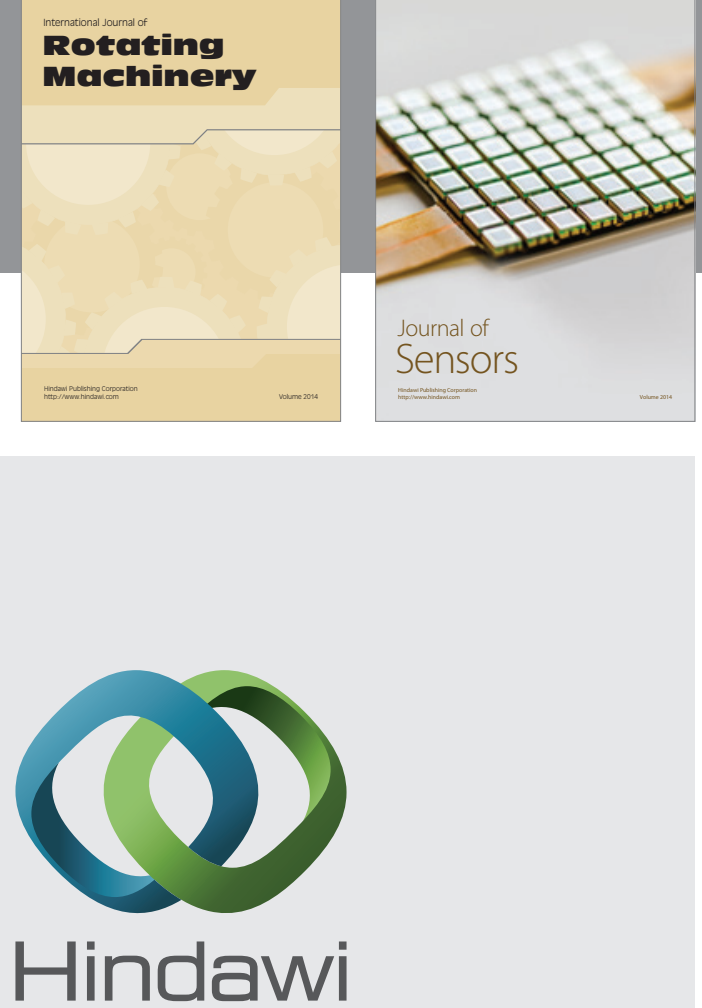

Submit your manuscripts at http://www.hindawi.com
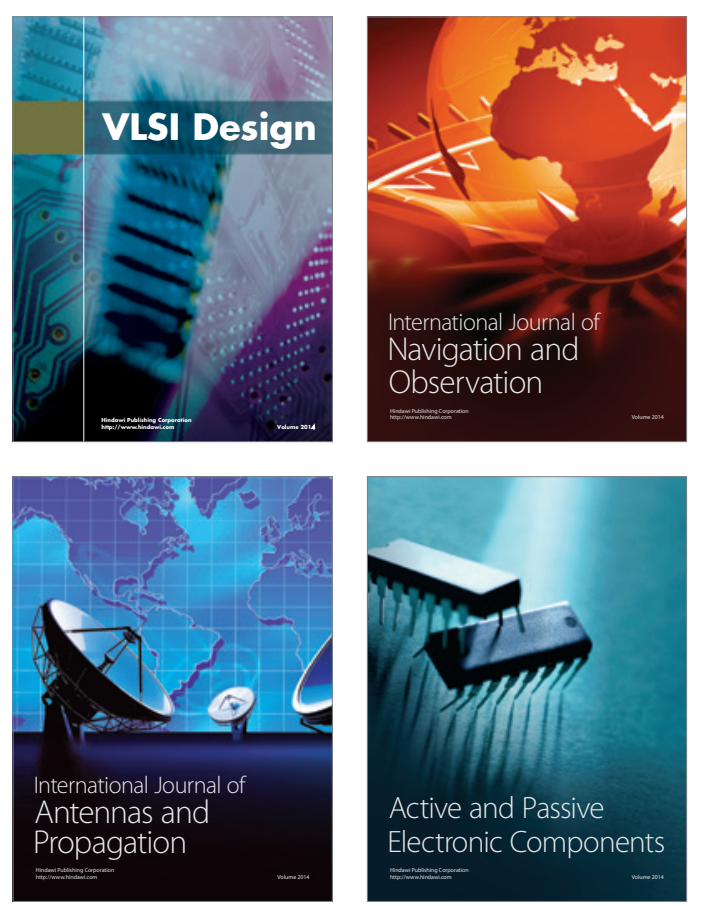
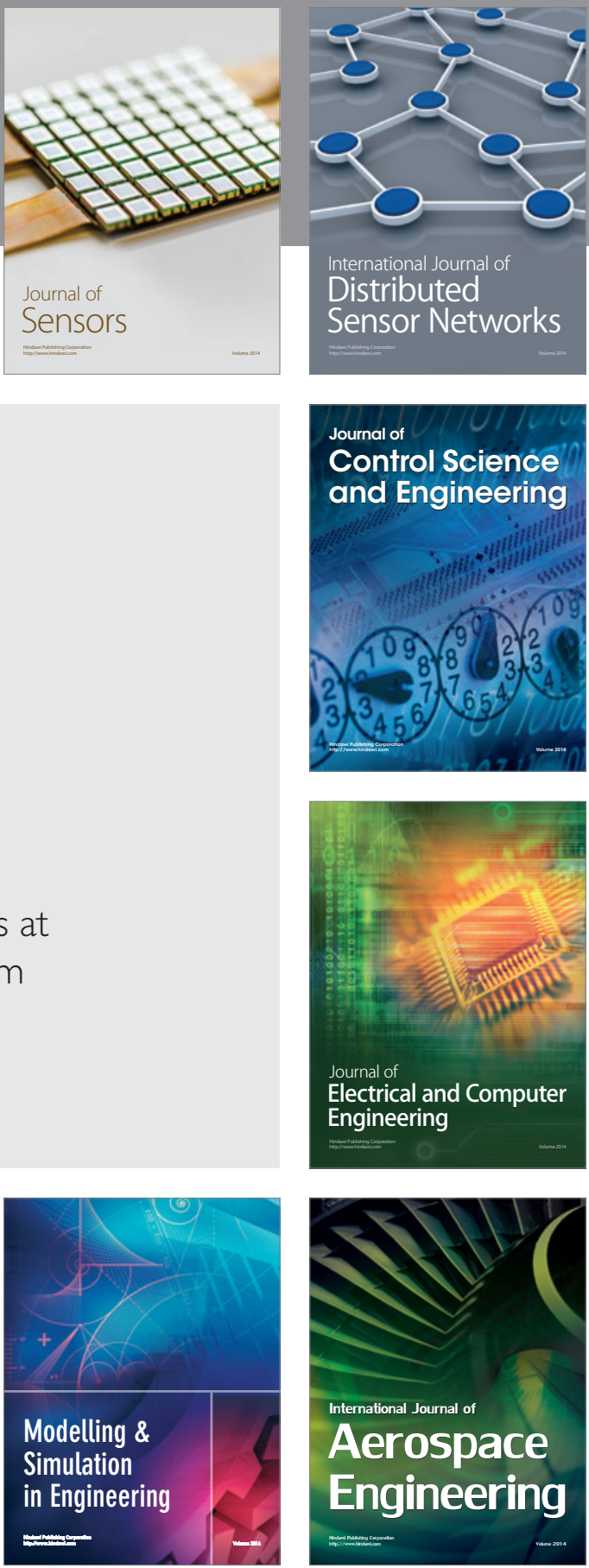

Journal of

Control Science

and Engineering
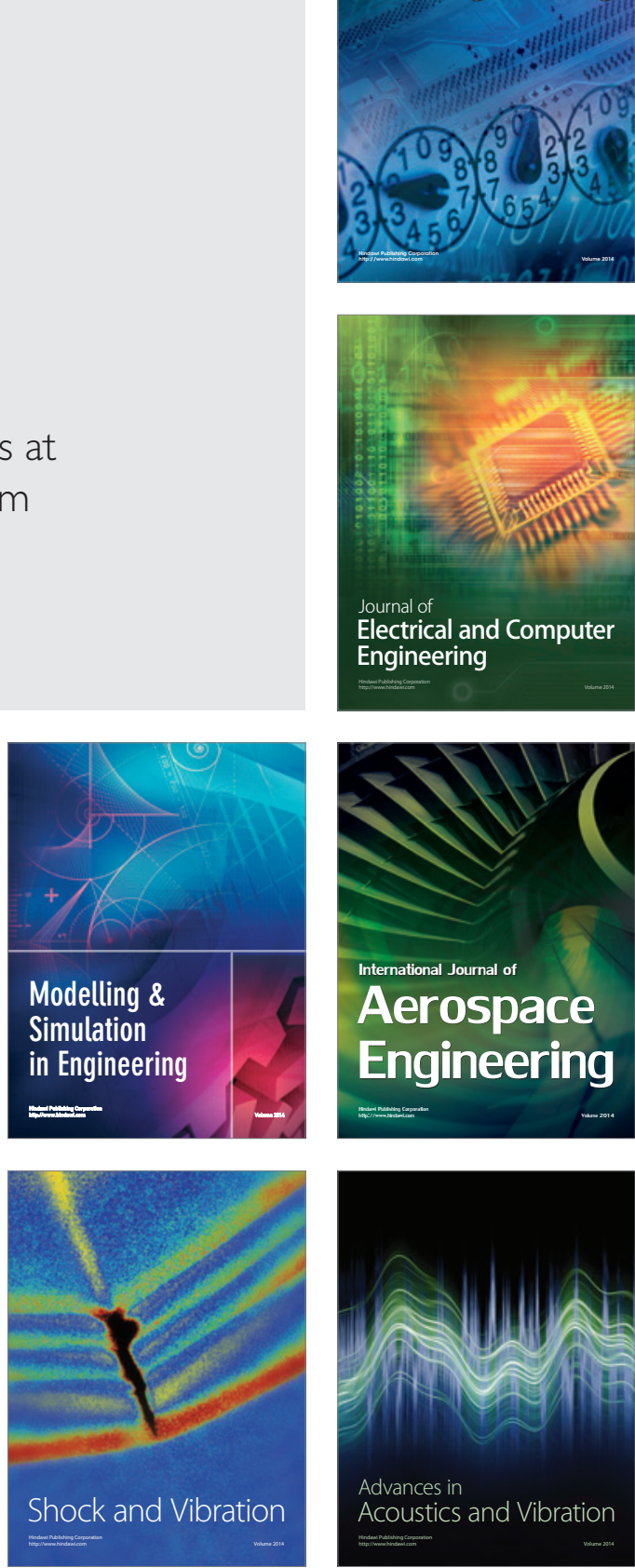